University of Nebraska - Lincoln

DigitalCommons@University of Nebraska - Lincoln

6-15-1997

\title{
Solvent-induced interactions between hydrophobic and hydrophilic polyatomic sheets in water and hypothetical nonpolar water
}

\author{
Kenichiro Koga \\ University of Nebraska-Lincoln, koga@cc.okayama-u.ac.jp \\ Xiao Cheng Zeng \\ University of Nebraska-Lincoln, xzeng1@unl.edu \\ Hideki Tanaka \\ Kyoto University, htanakaa@cc.okayama-u.ac.jp
}

Follow this and additional works at: https://digitalcommons.unl.edu/chemzeng

Part of the Chemistry Commons

Koga, Kenichiro; Zeng, Xiao Cheng; and Tanaka, Hideki, "Solvent-induced interactions between hydrophobic and hydrophilic polyatomic sheets in water and hypothetical nonpolar water" (1997). Xiao Cheng Zeng Publications. 70.

https://digitalcommons.unl.edu/chemzeng/70

This Article is brought to you for free and open access by the Published Research - Department of Chemistry at DigitalCommons@University of Nebraska - Lincoln. It has been accepted for inclusion in Xiao Cheng Zeng Publications by an authorized administrator of DigitalCommons@University of Nebraska - Lincoln. 


\title{
Solvent-induced interactions between hydrophobic and hydrophilic polyatomic sheets in water and hypothetical nonpolar water
}

\author{
Kenichiro Koga and X. C. Zeng \\ Chemistry Department and Center for Materials Research and Analysis, University of Nebraska-Lincoln, \\ Lincoln, Nebraska 68588 \\ Hideki Tanaka \\ Department of Polymer Chemistry, Kyoto University, Kyoto 606-01, Japan
}

(Received 7 January 1997; accepted 12 March 1997)

\begin{abstract}
Hydrophobic and hydrophilic interactions are two major intermolecular forces between hydrophobic nonpolar and hydrophilic polar sites of macromolecules or materials surfaces in solvents. To further understand these two interactions at the microscopic level, an idealized polyatomic model is devised, which includes hydrophobic, hydrophilic, and partially hydrophilic polyatomic planar square molecular sheets. The hydrophobic molecular sheet is composed of the Lennard-Jones particles while the hydrophilic molecular sheet consists of positive and negative charge sites. In the framework of the extended reference interaction site model integral equation theory the solvent-induced interactions (or the potential of mean forces) between two parallel molecular sheets in water and in the hypothetical nonpolar water are investigated in a systematic fashion. Such a highly idealized model allows us to isolate and to explore the important effects of molecular size, relative intermolecular position (e.g., in- or out-of-registry configuration), and hydrophilic site distribution on the hydrophobic and hydrophilic interactions in both water and the hypothetical nonpolar water. Significant insight into these effects at the molecular level is obtained. For the hydrophobic planar molecules in water we find solvent separated hydrophobic interaction becomes less favored as sheet size increases. Moreover, the contact hydrophobic interaction between two molecular sheets in the out-of-registry configuration is always most favorable. For the latter case we find it is the van der Waals attraction, rather than the hydrophobic attraction, that dominates the total interaction. We also find that in both water and the hypothetical nonpolar water the solvent-induced interaction between two hydrophobic sheets behaves similarly. One possible explanation is that the hydrophobic hydration originating from the hydrogen bonding network in water plays an insignificant role in the solvent-induced interaction, at least in the infinitely dilute aqueous solution. For hydrophilic planar molecular sheets in water, we find water-induced hydrophilic interaction is much more substantial compared with the hydrophobic one. In many cases, the hydrophilic interaction is found directly against the intermolecular force between two parallel molecular sheets in vacuum. Finally, for the partially hydrophilic planar molecules in water, a newly discovered feature is that a disperse hydrophilic site distribution gives rise to stronger solvent-induced interaction compared with the clustered hydrophilic site distribution. (C) 1997 American Institute of Physics. [S0021-9606(97)51123-8]
\end{abstract}

\section{INTRODUCTION}

Since many physical association processes such as the folding of proteins, binding of substrates to enzymes, and formation of micelles are carried out only in aqueous environments, these processes evince that interactions between macromolecules or materials surfaces containing hydrophilic polar and hydrophobic nonpolar sites or groups in water are distinctive, compared with those in vacuum and other solvents. ${ }^{1-6}$ However, behaviors of these interactions in water are still not fully understood at the molecular level. Without such an understanding of the effect of water on the interor intra-molecular interactions, the structural and dynamical properties of macromolecules in aqueous solutions as well as forces between materials surfaces in water cannot be accurately characterized and predicted. Because of the complexity of macromolecules or materials surfaces, however, traditionally effects of water were treated via a continuum media with a given dielectric constant. Recently, the development of fast computer and computational methodology has greatly expanded the scope of computer simulations into the explicit treatment of solvent surrounding macromolecules such as proteins and nucleic acids. ${ }^{3-5,7}$ Even so, calculation of the solvent-induced properties (e.g., entropy of hydrophobic hydration $^{8}$ ) for complex molecules still demands extremely long computer time.

An alternative approach for the study of the solventinduced interactions between complex molecules is to use the extended reference interaction site model (RISM) integral equation theory, ${ }^{9,10}$ which is a renormalized form of the RISM equation ${ }^{11}$ with the hypernetted chain (HNC) analogue closure. An advantage of the integral equation approach to computer simulation is that one can obtain the pair correlation functions free from statistical errors with much less computational effort. Furthermore, since the extended 
RISM theory is capable of dealing with polyatomic molecules interacting with both short- and long-range site-site pair potentials, application of this theory is beyond simple molecular fluids. It should be noted that because of the inherent approximation in the integral equation, ${ }^{12}$ in certain cases the extended RISM equation theory may generate significant errors. For example, Chen and Rossky ${ }^{13}$ found that in the case of a solute atom approaching a group of atoms, interaction between them is only qualitatively accounted for. We also find the calculated pressure of the TIPS water is much higher than the experimental value (See Sec. IV A). Therefore, the extended RISM theory is most suitable for gaining molecular insight based on semiquantitative analysis and for serving as a complementary means to computer simulations.

Solvent effects on the interactions between two molecules can be examined by evaluation of the potentials of mean force (PMFs) or, more precisely, the solvent-induced term of the PMFs. Many attempts to evaluate the PMFs (or equivalently the pair correlation functions) using computer simulations and integral equation theory have been performed for simple hydrophobic solutes, ${ }^{14-26,8,27-30}$ ionic solutes, ${ }^{31-37,13}$ and polar solutes ${ }^{38,39}$ in dilute aqueous solutions. In some cases quantitative or qualitative differences among results on the PMFs were found for certain systems. (For example, there is no agreement as to whether the PMF of $\mathrm{Na}^{+}-\mathrm{Cl}^{-}$in dilute aqueous solution has a second minimum in addition to the contact minimum. ${ }^{31-36}$ ) This is because the PMFs are determined by adding two competing terms: the direct interaction in vacuum and the solventinduced interaction. Despite the subtlety to obtain exact PMFs, many theoretical approaches have predicted the same trend for the PMFs. For example, both the extended RISM and computer simulations reveal the presence of a local minimum in the PMF for anionic pairs near contact ${ }^{34,37}$ (which cannot be predicted from dielectric continuum models). Another trend is the strong solvent-induced interaction between polar molecules at certain configurations. For example, the PMF for a pair of acetonitrile molecules in water shows a stable minimum in a relative configuration such that two negatively charged nitrogen atoms are nearly in contact with each other. ${ }^{38}$ Results of molecular dynamics (MD) simulations have shown that the solvent-induced force between a pair of water molecules as solute in water can be much stronger at certain separations than that for a pair of nonpolar molecules. ${ }^{39}$

Although great progress has been made in the study of the interionic and intermolecular PMFs in dilute aqueous solutions, current knowledge on the PMFs is still limited to the cases of simple molecules. Only solvation free energies and intramolecular PMF surfaces for rather complex biological molecules such as dipeptides ${ }^{40-42}$ have been studied. The results of the PMF surfaces in solution were found to be very different in character from those in vacuum and even counter-intuitive for hydrophilic groups. ${ }^{40,41}$ Recently BenNaim suggested ${ }^{43}$ that under favorable conditions, solventinduced forces between large molecules containing hydrophilic sites can be stronger and longer-ranged than between two small hydrophilic molecules. If so, these solvent-induced interactions, other than the hydrophobic interactions, would play a decisive role in many physical association processes, ranging from protein-protein association to interaction between hydrophilic clay minerals.

In order to further our understanding of the solventinduced interactions for more complex macromolecules and materials surfaces, it is sensible to explore the hydrophobic and hydrophilic interactions by using idealized models in some systematic fashion. To this end we devised an idealized polyatomic planar sheet model in order to investigate the hydrophobic and hydrophilic interactions as a function of sheet size, relative molecular position, strength of hydrophilicity, as well as distribution of hydrophilic sites on the molecular sheet.

Previous theoretical and computational studies of this kind have brought about several important findings on hydrophobic interactions between spherical solutes. ${ }^{14-26,8,27-30}$ In this work, we employ the extended RISM theory to examine the solvent-induced interactions between two polyatomic planar sheets with hydrophobic or hydrophilic sites. Atomic sites on the hydrophobic molecular sheet hold no charge while those on the hydrophilic molecular sheet possess negative or positive charge. Solvent-induced PMF is obtained through the solvation free energy calculation for a pair of molecular sheets in fixed configurations. ${ }^{13,38}$

Section II outlines the extended RISM theory, methodology for solvation free energy calculation, and the relation between the PMF and the solvation free energy. Section III introduces models of water, the hypothetical nonpolar water, and the molecular sheet with hydrophobic or hydrophilic sites. Calculation results and discussion are given in Sec. IV. Section V presents our concluding remarks.

\section{THEORY}

\section{A. Potential of mean force}

The potential of mean force (PMF) between two molecules $\alpha$ and $\beta$ in a solvent, $W(\mathbf{R})$, is the work for bringing the molecular pair at infinite separation to a relative configuration $\mathbf{R}$, where $\mathbf{R}$ denotes the relative position as well as orientation of the two molecules. The work is equivalent to the Helmholtz (Gibbs) free energy change of the process under constant NVT (NPT) condition. Thus the PMF can be written as

$$
W(\mathbf{R})=U(\mathbf{R})+\delta A(\mathbf{R}),
$$

where $U(\mathbf{R})$ is the direct pair potential function in the configuration $\mathbf{R}$ and $\delta A(\mathbf{R})$ is the indirect free energy change induced by solvent in the above process. $\delta A(\mathbf{R})$ in Eq. (1) can be further expressed as a difference between the solvation free energy ${ }^{44}$ (or the excess chemical potential), $\Delta A^{*}$, of the pair in the relative configuration $\mathbf{R}$ and that at infinite separation,

$$
\begin{aligned}
\delta A(\mathbf{R}) & =\Delta A_{\alpha \beta}^{*}(\mathbf{R})-\Delta A_{\alpha \beta}^{*}(\infty) \\
& =\Delta A_{\alpha \beta}^{*}(\mathbf{R})-\Delta A_{\alpha}^{*}-\Delta A_{\beta}^{*},
\end{aligned}
$$


where the second line results from the fact that the solvation free energy of the pair at infinite separation may be viewed as the sum of the solvation free energies of single molecule $\alpha$ and $\beta$. The solvation free energy for molecules constructed by interaction sites can be evaluated in the framework of the extended RISM theory (Sec. II B). One can view a pair of molecules $\alpha$ and $\beta$ in the relative configuration $\mathbf{R}$ as a single supermolecule $\alpha-\beta$ in a fixed intra-supermolecular configuration $\mathbf{R} . \delta A(\mathbf{R})$ and the corresponding PMF between $\alpha$ and $\beta$ molecules in solvent can then be determined using a so-called supermolecular approach illustrated below. Note that the first term on the right-hand side of Eq. (2) can be written as a sum of two terms: the solvation free energy of $\beta, \Delta A_{\beta}^{*}$, and the conditional solvation free energy of $\alpha$ at a fixed position $\mathbf{R}, \Delta A_{\alpha / \beta}^{*}(\mathbf{R})$ (where $\beta$ is fixed at the origin of the coordinate in the solvent ${ }^{44}$ ). Equation (2) can then be written as

$$
\delta A(\mathbf{R})=\Delta A_{\alpha / \beta}^{*}(\mathbf{R})-\Delta A_{\alpha}^{*} .
$$

Clearly one can exchange the suffix $\alpha$ and $\beta$ in Eq. (3). The latter equation also shows how the solvation free energy of $\alpha(\beta)$ is affected by the existence of the molecule $\beta(\alpha)$ in the relative configuration $\mathbf{R}$. Note also that the PMF is related to the pair distribution function, $g(\mathbf{R})$ : $\beta W(\mathbf{R})=-\ln g(\mathbf{R})$, where $\beta=\left(k_{B} T\right)^{-1}, k_{B}$ is the Boltzmann constant. Once the PMF is known, the corresponding force between the pair molecules can be determined from

$$
F(\mathbf{R})=-\nabla U(\mathbf{R})-\nabla \delta A(\mathbf{R}),
$$

where $\nabla$ is the gradient with respect to the vector $\mathbf{R}$. The first term on the right-hand side of Eq. (4) is the direct force in vacuum independent of the existence of solvent molecules, and the second term is the indirect or solvent-induced force.

\section{B. The extended RISM equation}

In this section we outline the extended RISM equation theory. Details of the theory are described in the literature. .,10,45,46 Essentially, the RISM equation $^{11}$ is an Ornstein-Zernike-like relation between the site-site pair correlation functions $h_{i j}$ and the direct correlation functions $c_{i j}$

$$
\mathbf{h}=\mathbf{w c w}+\mathbf{w c \rho h},
$$

where the matrix elements of $\mathbf{h}$ and $\mathbf{c}$ are the functions $h_{i j}$ and $c_{i j}$ in Fourier $k$ space, respectively. $\boldsymbol{\rho}$ is a diagonal matrix whose elements are number densities of each molecular species, and $\mathbf{w}$ is an intramolecular correlation matrix. The extended RISM equation is a renormalized form of the original RISM equation for the treatment of the site-site pair potentials $u_{i j}$ containing long-range parts $-\beta^{-1} \phi_{i j} \cdot{ }^{9,10}$ Using the renormalized potential matrix,

$$
\mathbf{Q}=\mathbf{w} \boldsymbol{\phi}[\mathbf{I}-\boldsymbol{\rho} \mathbf{w} \boldsymbol{\phi}]^{-1} \mathbf{w}
$$

and the short-range direct correlation matrix $\mathbf{c}_{\boldsymbol{s}} \equiv \mathbf{c}-\boldsymbol{\phi}$, the extended RISM equation is given by

$$
\boldsymbol{\tau}=\{\mathbf{w}+\mathbf{Q} \boldsymbol{\rho}\} \mathbf{c}_{\mathbf{s}}\{\mathbf{w}+\boldsymbol{\rho h}\}-\mathbf{c}_{\mathbf{s}},
$$

where $\boldsymbol{\tau} \equiv \mathbf{h}-\mathbf{c}-\mathbf{Q}$. Equation (7) can be solved using the standard iterative method under the site-site hypernetted chain (HNC) analogue closure approximation,

$$
c_{s i j}=\exp \left(-\beta u_{i j}^{0}+\tau_{i j}+Q_{i j}\right)-\tau_{i j}-Q_{i j}-1,
$$

where $u_{i j}^{0}=u_{i j}+\beta^{-1} \phi_{i j}$ is the short-range part of the sitesite pair potential functions.

Within the context of the extended RISM theory in conjunction with the HNC analogue closure equation, Singer and Chandler ${ }^{45}$ have shown that the solvation free energy of a solute molecule at infinite dilution can be written in an analytical form and without integration over a coupling parameter $\lambda$. This solvation free energy expression is ${ }^{46}$

$$
\begin{aligned}
\beta \Delta A^{*}= & 4 \pi \rho \sum_{i j} \int d r r^{2}\left\{\frac{1}{2}\left[h_{i j}^{\mathrm{vu}}(r)\right]^{2}-c_{i j}^{\mathrm{vu}}(r)\right. \\
& \left.-\frac{1}{2} h_{i j}^{\mathrm{vu}}(r) c_{i j}^{\mathrm{vu}}(r)\right\},
\end{aligned}
$$

where $h^{\mathrm{vu}}$ and $c^{\mathrm{vu}}$ denote the site-site pair and direct correlation functions between solvent and solute molecules. By evaluating the solvation free energies, using Eq. (9), of each single molecule $\alpha$ and $\beta$ as well as the supermolecule $\alpha-$ $\beta$ in a fixed relative configuration in solvent, the solventinduced part of the PMF in that relative configuration can be obtained. The significance of this supermolecule approach (proposed independently by Chen et al. ${ }^{13}$ and Matsumoto et $a l^{38}$ ) is that one can calculate the full functions of the PMF (or equivalently the full pair distribution functions) including the detailed information of orientation dependence, whereas the conventional RISM approach can only give the site-site radial distribution functions in which the information on orientation dependence in the full distribution functions is averaged out. Also note that no further approximation to the extended RISM theory is involved in the supermolecule approach. It has been shown that for nonpolar, polar, and ionic solutes intramolecular and intermolecular free energy surfaces in solvents obtained from the supermolecule approach ${ }^{13,38}$ or the coupling parameter scheme ${ }^{46}$ are in very good agreement with computer simulation and experimental results.

\section{THE MODEL SYSTEM}

The polyatomic planar molecular sheets are constructed with the same structure as a square portion of the $(1,0,0)$ surface of the NaCl-type crystal. Distance between nearestneighbor atomic sites on the sheet, $l$, is $2^{1 / 6} \sigma_{a}$, where $\sigma_{a}$ is the Lennard-Jones (LJ) parameter of interatomic interaction. Total interaction between two molecular sheets (1 and 2) in the relative configuration $\mathbf{R}$ is expressed as

$$
U(\mathbf{R})=\sum_{i, j=1}^{n_{a}} 4 \epsilon\left[\left(\sigma_{a} / r_{i j}\right)^{12}-\left(\sigma_{a} / r_{i j}\right)^{6}\right]+Z_{i} Z_{j} / r_{i j},
$$

where $n_{a}$ is the number of atomic sites in one molecular sheet, $r_{i j}$ is the distance between the $i$ th atomic site in sheet 1 and $j$ th atomic site in sheet 2 , and $Z_{i}$ is the magnitude of 


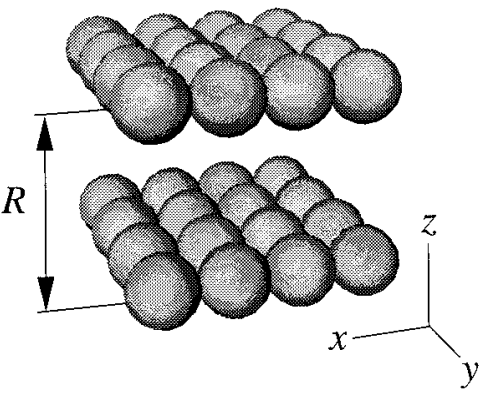

FIG. 1. Two molecular sheets in a parallel in-registry configuration. $R$ is the intersheet distance.

partial charge of the $i$ th atomic site. The LJ parameters $\sigma_{a}$ and $\epsilon / k_{B}$ are $3.395 \AA$, and $67.1 \mathrm{~K}$, which are those for atomic sites of naphthalene. ${ }^{47}$

The hydrophobic molecular sheets bear no partial charge on any atomic site. For the hydrophilic molecular sheets each atomic site has a partial charge $Z$ or $-Z$, which is placed reciprocally as that in the $(1,0,0)$ plane of the $\mathrm{NaCl}$ crystal. All the results for the hydrophilic molecular sheets are obtained by setting the magnitude of the charge $|Z|$ to $0.8 e$ unless otherwise mentioned.

To examine effects of sheet size on the PMF between two molecular sheets, five sheet sizes, $n_{a}=1,4,9,16$, and 25 were used in this study. (Note that in the case of $n_{a}=1$ the solute is no longer a sheet but a spheric particle.) For the hydrophilic molecular sheets, only $n_{a}=4$ and $n_{a}=16$ sheet sizes were used.

The PMF is obtained as a function of $R$, the separation between two parallel planar molecular sheets. Obviously there are unlimited choices of relative configuration of the two sheets. Here two particular configurations were chosen: one is a configuration in which atomic sites of two sheets are completely in registry (the in-registry configuration), and the other is a configuration in which atomic sites are completely out of registry (the out-of-registry configuration). The two configurations differ from each other by a half of $l$ in both the $x$ and $y$ directions (see Fig. 1). Most calculations in this study were devoted to the in-registry configuration because it demands the least computational effort due to the intersheet site-position symmetry. For hydrophobic molecular sheets, both in-registry and out-of-registry configurations were considered. For hydrophilic molecular sheets the relative configuration was always maintained to be in registry whereas two types of charge distribution on the molecular sheets (described in Sec. IV as charge symmetric and asymmetric configurations) were considered. Figure 1 presents a pair of $4 \times 4$ sheets $\left(n_{a}=16\right)$ in the in-registry configuration.

Two types of solvent are considered in this study, water and the hypothetical nonpolar water. The water model adopted here is the simple three-site TIPS model ${ }^{48}$ of Jorgensen with slight modification. ${ }^{49}$ The hypothetical nonpolar water is constructed by setting all partial charges of the TIPS water to be zero. The molecular sheet-water potential function is also expressed by a sum of site-site potential functions, $u_{i j}^{\mathrm{vu}}$, which consists of the LJ and the Coulombic

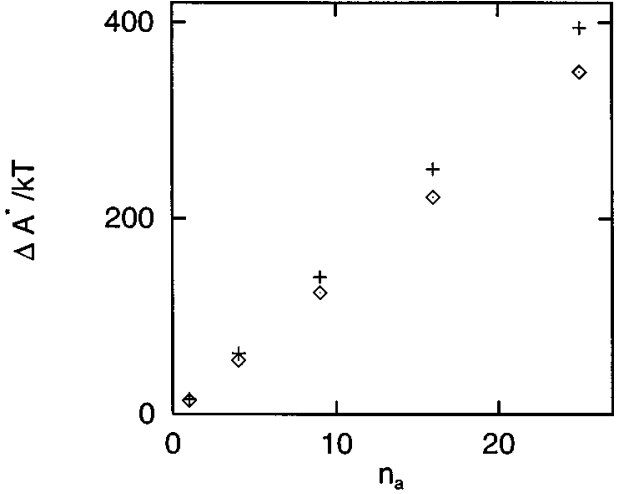

FIG. 2. The solvation free energy $\Delta A^{*}$ of a hydrophobic molecular sheet in water $(\diamond)$ and in the hypothetical nonpolar water $(+)$ at $298.15 \mathrm{~K}$ as a function of the number of atomic site $n_{a}$ on the sheet.

terms. The LJ parameters $\left(\sigma_{w a}, \epsilon_{w a}\right)$ are given by the Lorentz-Berthelot rule: $\sigma_{w a}=\left(\sigma_{w}+\sigma_{a}\right) / 2, \quad \epsilon_{w a}=\sqrt{\epsilon_{w} \epsilon_{a}}$, where $\sigma_{w}$ and $\epsilon_{w}$ denote the LJ parameters for the solventsolvent interaction. All the calculations were performed at a temperature of $297.15 \mathrm{~K}$ and solvent number density $0.033456 \AA^{-3}$, which corresponds to $1.0 \mathrm{~g} / \mathrm{cm}^{3}$ for water.

\section{RESULTS AND DISCUSSION}

\section{A. Solvation free energy of single hydrophobic sheet}

Before discussing the hydrophobic interaction between two hydrophobic molecular sheets in water, we first examine sheet-size dependence of the solvation free energy for a single hydrophobic molecular sheet, i.e., the so-called hydrophobic hydration effect, and compare the results with experiments for hydrocarbons. Figure 2 shows calculated $\Delta A^{*}$ versus the size of the hydrophobic molecular sheet $\left(n_{a}=1,4,9\right.$, 16 , and 25). We find that $\Delta A^{*}$ of a single molecular sheet in both TIPS and the hypothetical water changes linearly with sheet-size $n_{a}$. We also notice that a linearity of the solvation free energy for homologous series of hydrocarbons in water $^{50,51}$ has been observed experimentally. Thus the extended RISM theory correctly predicts the trend for the solvation free energy of hydrocarbon in water. This prediction is significant because it provides important evidence that the extended RISM theory affords qualitatively correct results on solvation properties of complex molecules. On the other hand our results show that a linearity of $\Delta A^{*}$ as a function of $n_{a}$ holds for both water and the hypothetical nonpolar water. This suggests that water is not so special as far as the linear dependence of the solvation free energy on molecular size is considered. As to the absolute values of the free energy (Fig. 2 ), it seems counter-intuitive that $\Delta A^{*}$ for the hypothetical nonpolar water is systematically slightly higher than the corresponding $\Delta A^{*}$ for the TIPS water. We should note, however, that in this study the density of both types of solvent, but not the pressure $P$, was set to be the same. In fact, we find that under the same density, the experimental values of $P$ for water and that for the $\mathrm{LJ}$ fluid $^{52}$ (using the well-known equation of state of the LJ liquid ${ }^{53}$ and the same LJ parameters as the TIPS water model) are very different: for water 

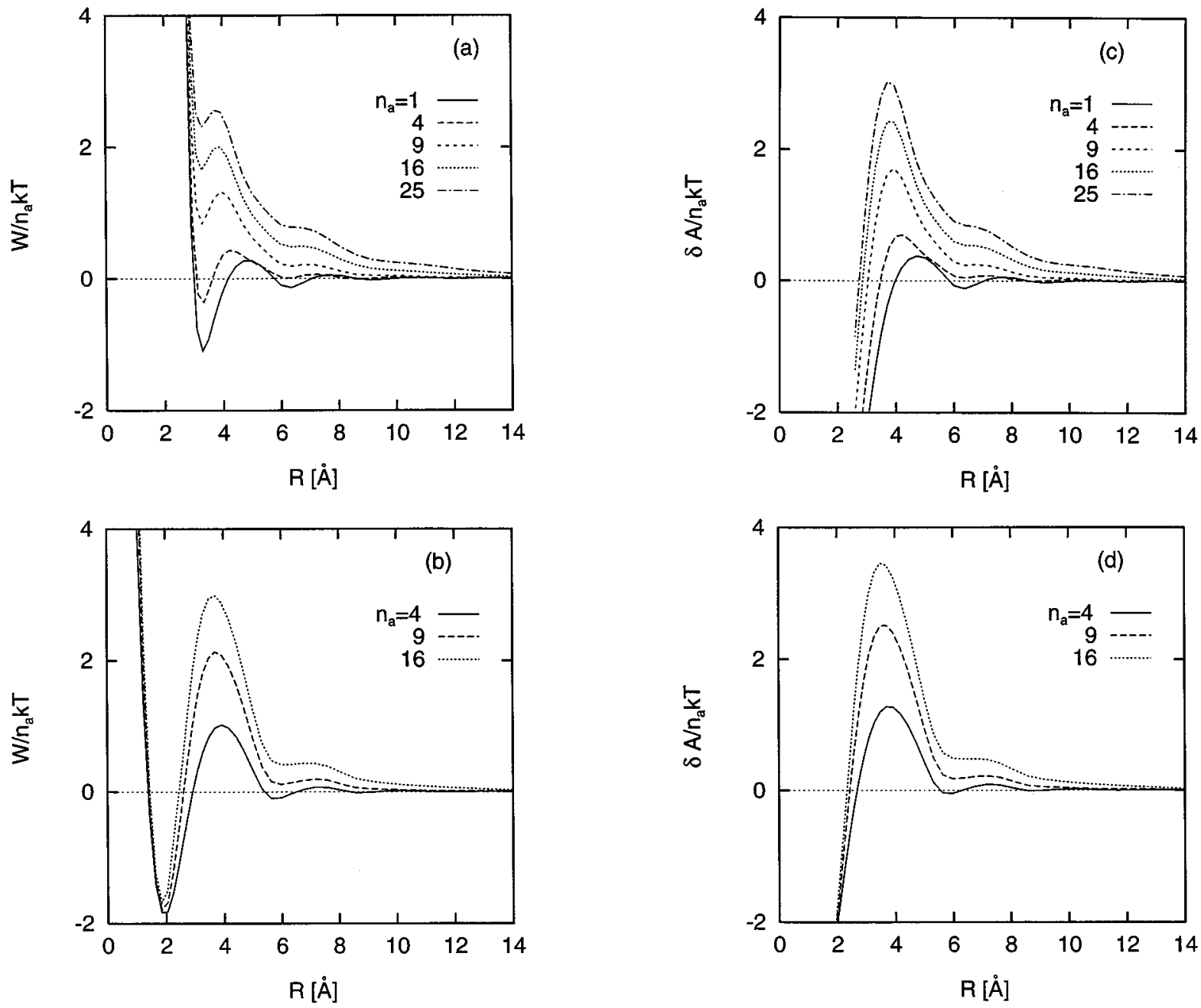

FIG. 3. The intersheet interactions per atomic site between two hydrophobic molecular sheets with five different sizes $n_{a}$. (a) The PMFs for in-registry pairs; (b) the PMFs for out-of-registry pairs; (c) the solvent-induced interactions for in-registry pairs; and (d) the solvent-induced interactions for out-of-registry pairs. $T$ is the temperature of the solvent.

$P=0.1 \mathrm{MPa}\left(0.00073 \rho k_{B} T\right)$ and for the LJ fluid, $P=1200$ $\mathrm{MPa}\left(8.74 \rho k_{B} T\right)$. In contrast, pressure calculated using the extended RISM theory via the compressibility route ${ }^{12}$ is 449 $\mathrm{MPa}\left(3.28 \rho k_{B} T\right)$ for the TIPS water and $759 \mathrm{MPa}$ $\left(5.54 \rho k_{B} T\right)$ for the hypothetical nonpolar water, which is comparable. Therefore, it is possible that if both TIPS water and hypothetical nonpolar water are under the same pressure, one may find higher solvation free energy for the hypothetical nonpolar water than for the TIPS water.

\section{B. Solvent-induced interactions between two hydrophobic sheets}

PMF per atomic site between two parallel hydrophobic molecular sheets (in the in-registry configuration) in water are plotted in Fig. 3(a). Clearly the PMF per atomic site is sensitive to the sheet size. Only if the sheets are very small $\left(n_{a} \leqslant 4\right)$ is the pair of contacting sheets thermodynamically stable in the in-registry configuration. For larger sheet sizes $\left(n_{a} \geqslant 9\right)$, the contacting configurations are metastable; and as the sheet size becomes larger the potential barrier separating the contacting configuration and the solvent-separated con- figuration becomes higher. However this result does not mean that a pair of large hydrophobic molecular sheets never associate with each other when dissolved in water. The most stable geometry of two hydrophobic molecular sheets can only be obtained when the full PMF surface is known. Figure 3(b) shows the PMF per atomic site for an out-of-registry pair of hydrophobic molecular sheets in water. In this configuration, we find a contacting pair is thermodynamically stable regardless of the size of the sheets; the minimum in the PMF curves (corresponding to the contacting pair) is located around $2 \AA$. The large difference in the PMF between the in-registry and out-of-registry pair is mainly attributed to the difference in the direct interaction between two molecular sheets in vacuum. On the other hand, a common feature found in both cases is that the barrier separating the contacting configuration from the solvent-separated configuration becomes higher when sheet-size $n_{a}$ is larger. This common feature is mainly due to the similar behavior of the size dependence of the solvent-induced interactions $\delta A$ per atomic site as shown in Figs. 3(c) and 3(d).

Further insight can be gained when the solvent-induced 

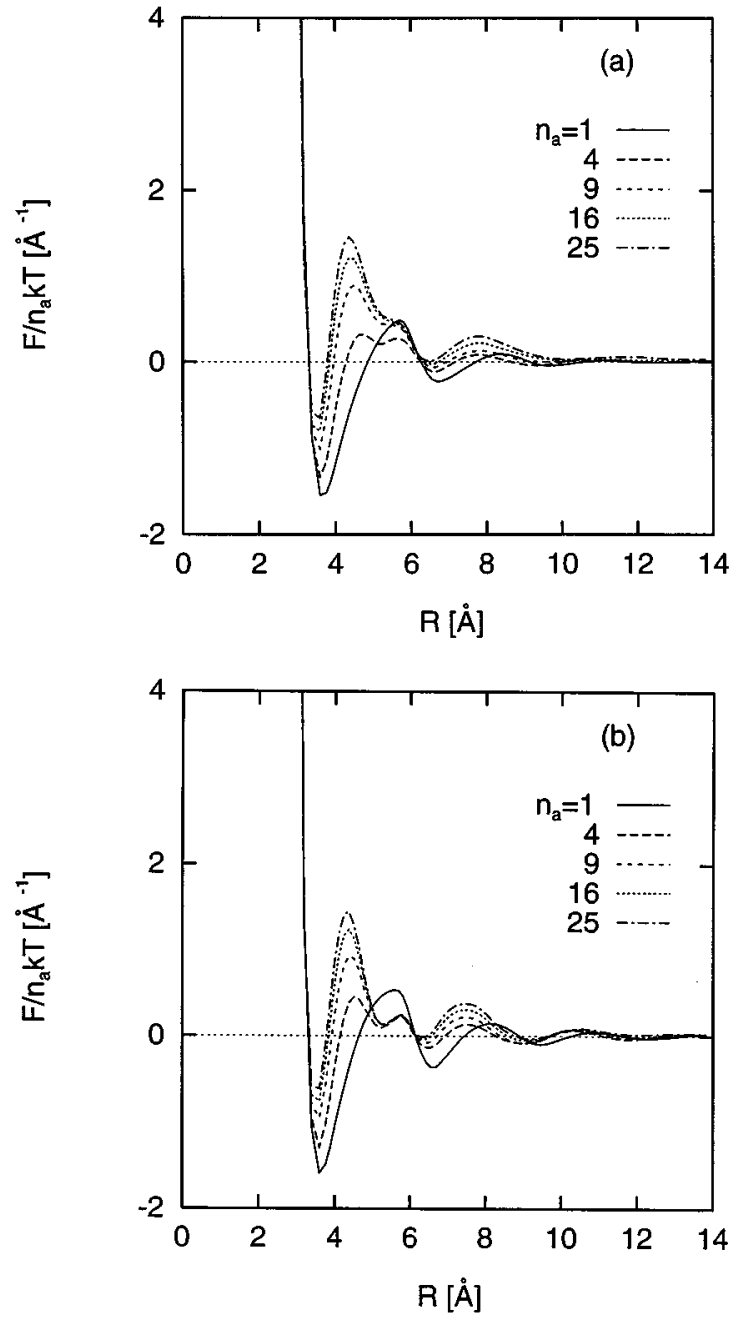

FIG. 4. The mean forces per atomic site between two hydrophobic molecular sheets: (a) in water; (b) in the hypothetical nonpolar water.

interaction per atomic site $\delta a\left(R \mid n_{a}\right)$ is expressed as a sum of the following two terms:

$$
\delta a\left(R \mid n_{a}\right)=\Delta A_{d}^{*}(R) / n_{a}-2 \Delta A_{s}^{*} / n_{a},
$$

where $\Delta A_{d}^{*}(R)$ is the solvation free energy of the whole molecular pair (the supermolecule) with separation distance $R$ and $\Delta A_{s}^{*}$ is the single molecular sheet solvation free energy. Since both $\Delta A_{d}^{*}$ and $\Delta A_{s}^{*}$ are linear with $n_{a}$, i.e., $\Delta A_{d}^{*}=k_{d} n_{a}+b_{d}$, and $\Delta A_{s}^{*}=k_{s} n_{a}+b_{s}$ (we have already mentioned in Sec. IV A that a linearity holds for a single sheet; we also have confirmed that this condition is satisfied for the pair at $R=2{ }^{1 / 6} \sigma_{a}$ ), then $\delta a$ can be expressed as

$$
\delta a\left(n_{a}\right)=\left(k_{d}-2 k_{s}\right)+\left(b_{d}-2 b_{s}\right) / n_{a} .
$$

Clearly Eq. (12) shows that as the sheet size $n_{a}$ increases $\delta a\left(n_{a}\right)$ increases if $b_{d}<2 b_{s}$ [as shown in Figs. 3(c) and $3(\mathrm{~d})$ ]. Indeed we find the latter condition is satisfied since $b_{d}$ is about $-20 k_{B} T$ at $R=2{ }^{1 / 6} \sigma_{a}$, while $2 b_{s}$ is nearly zero. Equation (12) also shows that for large $n_{a}, \delta a\left(n_{a}\right)$ becomes a constant, $k_{d}-2 k_{s}$.

Figure 4(a) shows the mean force between two inregistry molecular sheets versus intersheet distance. The mean force per atomic site is also found to be sensitive to the sheet size. As the sheet size increases, the repulsive force per atomic site becomes stronger around 4.5 and $8 \AA$. The force is almost zero around $6.5 \AA$ for all sizes of molecular sheet. Note that the latter distance is nearly the same as $2 \sigma_{w a}$ $(=6.6 \AA)$, the separation at which water molecules can just take the direction along the line connecting two facing atomic sites on two sheets. For comparison, the mean forces per atomic site in the hypothetical nonpolar water are given in Fig. 4(b); as the PMF, the mean force per atomic site shows similar behavior as that in water. A similar conclusion has been made from previous computer simulations. ${ }^{20,22,25,26}$ In fact, it seems to us that the hydrophobic attraction (either contact or solvent separated) can be explained as a reflection of the local liquid packing analogous to that shown in the pair distribution function of liquid between two hydrophobic walls. ${ }^{24,54}$

\section{Solvent-induced interactions between two hydrophilic sheets}

In this section we show the solvent-induced potentials between two parallel hydrophilic molecular sheets in water. It was pointed out by Ben-Naim and co-workers ${ }^{39,43}$ that under certain favorable conditions, hydrophilic interactions between molecules or molecular groups in water become much stronger than the corresponding hydrophobic interactions; the strong hydrophilic interactions originate in water hydrogen-bonded with the solute molecules or molecular groups. One favorable condition proposed ${ }^{39,43}$ is that negatively charged atomic sites of two molecules (or groups) face each other at a special separation such that a water molecule can form hydrogen bonds with the two molecules (or groups). To shed more light on the hydrophilic interaction we chose two configurations for a pair of hydrophilic molecular sheets: one is called the charge symmetric configuration in which signs of the partial charges of atomic sites on one sheet are the same as those on the opposite sheet (charges on two parallel sheets are in mirror symmetric configuration); the other is called the charge asymmetric configuration in which the signs of charge on two sheets are exactly opposite to each other. Note that in the charge symmetric configuration the Coulombic interaction is always repulsive, while in the charge asymmetric configuration the interaction is always attractive.

We first show the hydrophilic interaction between two molecular sheets in the charge symmetric configuration. Figure 5(a) displays the PMF between two $4 \times 4\left(n_{a}=16\right)$ parallel molecular sheets. As two sheets approach each other the PMF decreases monotonically up to distance about $3 \AA$ where it shows a low minimum with a depth about $120 k_{B} T$ (7.5 $k_{B} T$ per atom). The charge symmetric configuration becomes more stable as the intersheet distance decreases. This behavior of the PMF is completely different from that for the hydrophobic sheets. First, the magnitude of the hydrophilic interaction is much greater than that of the hydrophobic interaction; second, the PMF between two hydrophilic molecular sheets is negative except at very short distance, while the 

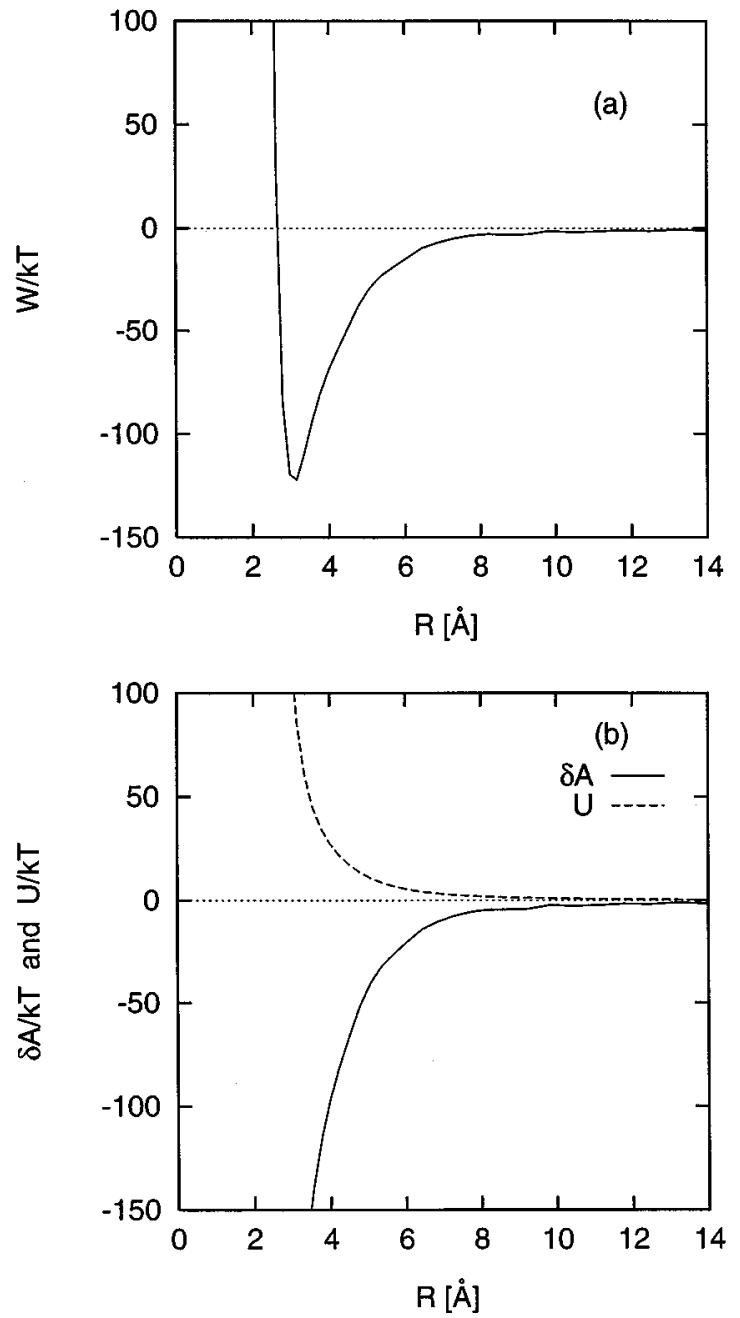

FIG. 5. The interactions between two hydrophilic molecular sheets $\left(n_{a}=16\right)$ in the charge symmetric configuration in water: (a) the PMF; (b) the solvent-induced interactions (solid line) and the direct interaction potential function in vacuum (dashed line).

PMF between two hydrophobic molecular sheets is positive at all separations. Figure 5(b) displays the direct interaction and solvent-induced interaction curves. These two curves reveal that the large negative PMF between two hydrophilic molecular sheets stems from the strong solvent-induced interaction which overwhelms the positive direct interaction in vacuum. Ben-Naim showed that the attractive forces between hydrophilic macromolecules would be strongly enhanced under the condition that the relative configuration of the two macromolecules allows water molecules to form hydrogen-bonding bridges in between. ${ }^{43}$ Here the charge symmetric configuration of the two hydrophilic molecular sheets is indeed a configuration that satisfies this condition.

For the charge asymmetric configuration, we find the PMF between two hydrophilic molecular sheets exhibits quite different features compared with those in the charge symmetric configuration. As shown in Fig. 6(a), the PMF is positive at all separations and monotonically increases as the distance between two sheets decreases. This is because, as shown in Fig. 6(b), the solvent-induced interaction $\delta A$ is
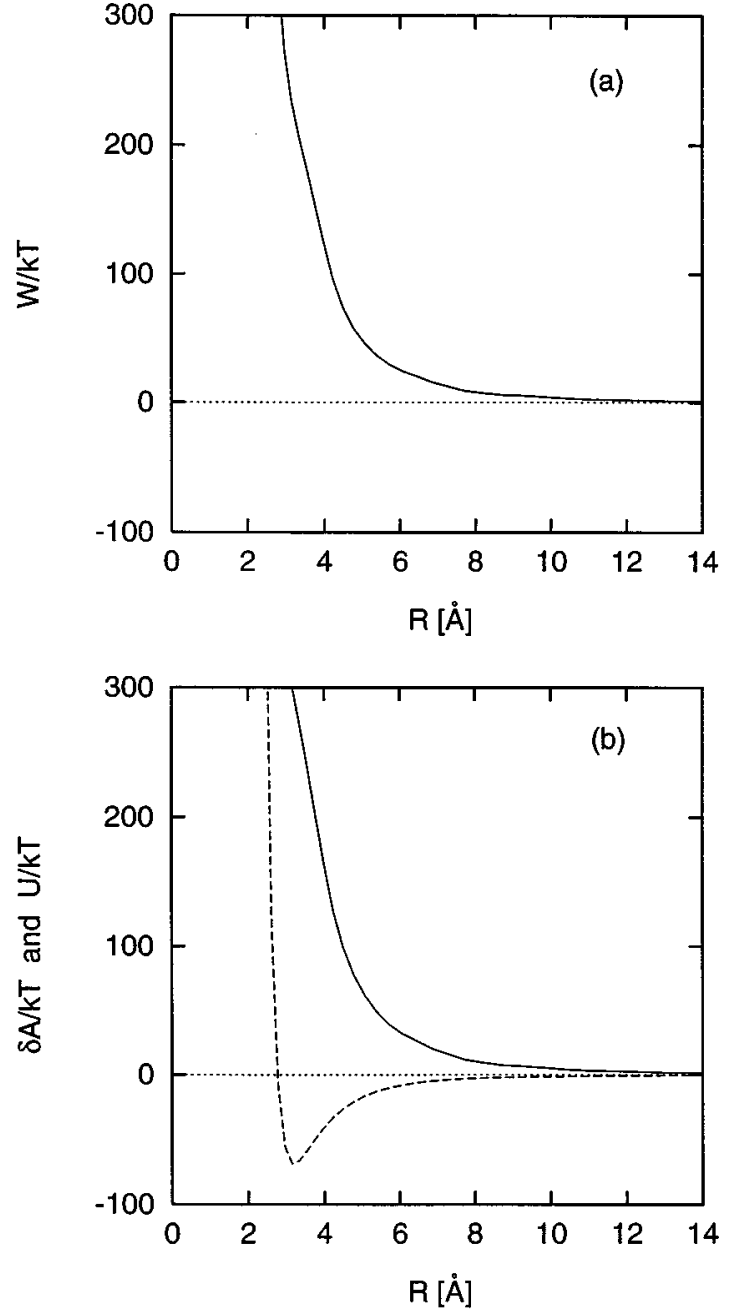

FIG. 6. The interactions between two hydrophilic molecular sheets $\left(n_{a}=16\right)$ in the charge asymmetric configuration in water: (a) the PMF; (b) the solvent-induced interactions (solid line) and the direct interaction potential function in vacuum (dashed line).

larger and also has an opposite trend to the direct interaction. In contrast to the charge symmetric configuration, the charge asymmetric configuration becomes more unstable as the distance between two sheets decreases.

Our results of the solvent-induced PMF between two hydrophilic molecular sheets lead to two important conclusions: First, interactions between two hydrophilic molecular sheets are much stronger in water than in vacuum; and are, in fact, against the direct interaction in vacuum. Second, the free energy surfaces as a function of relative configuration of the two hydrophilic molecular sheets would be much more rugged in water than those in vacuum, particularly for lateral $(x-y)$ directions when the distance between two sheets is fixed. We will argue later that the strong solvent-induced interaction is mainly due to the contribution of the $\delta E_{\mathrm{uv}}$ term in Eq. (13).

Next we show the dependence of the PMF on the strength of hydrophilicity of the molecular sheet. Figure 7 displays the solvent-induced terms of the PMF for three different pairs of $4 \times 4$ molecular sheets in the charge symmetric 


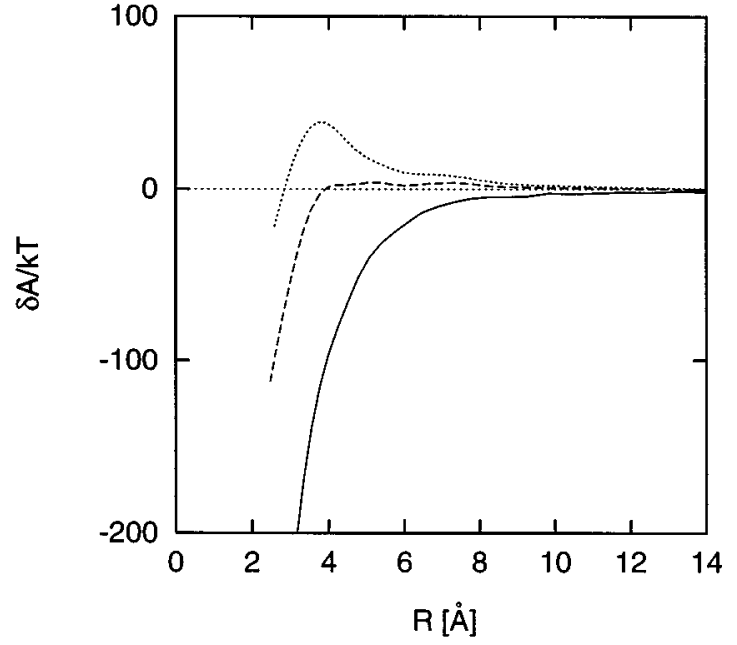

FIG. 7. The solvent-induced interactions between two hydrophilic molecular sheets $\left(n_{a}=16\right)$ in water for $|Z|=0 \mathrm{e}$ (dotted line), $0.4 \mathrm{e}$ (dashed line), and $0.8 \mathrm{e}$ (solid line). All pairs are in the charge symmetric configuration.

configuration (with charge $|Z| / e=0,0.4$, or 0.8 , respectively). It is found that as the magnitude of the partial charge increases the first peak appearing in the solvent-induced potential curve becomes smaller and eventually disappears, which suggests that the $\delta A-R$ relationship is very sensitive to the magnitude of the partial charge. This result also indicates that whether or not two hydrophilic molecular sheets take the contact configuration depends strongly on the magnitude of the charge of atomic site on the sheet. Strong dependence of $\delta A$ on the partial charge of the sheet is also found in the charge asymmetric configuration. It should be pointed out, however, that in the latter configuration the two sheets repel each other more strongly as the magnitude of the partial charge increases.

Finally, to show the sheet-size effect on the PMF we compared the PMFs of the $2 \times 2$ sheets with $4 \times 4$ sheets, both in the charge symmetric configuration. Figure 8(a) shows that the PMF per atomic site for the smaller sheets exhibits a deeper minimum than that for the larger ones. (The magnitude of the PMF itself between two larger sheets at the minimum is greater than that between two smaller sheets.) Since the direct repulsive interaction per atomic site is stronger for the smaller sheets than for the larger one, clearly, the deeper minimum in the PMF curve for the smaller sheets should be attributed to the $\delta A$ term. The latter term is more negative compared with that of the larger sheets [see Fig. 8(b)]. This trend of the sheet-size effect on $\delta A$ per atomic site is the same as that found for the hydrophobic molecular sheets; it is more noticeable, however, for the hydrophilic molecular sheets.

\section{Solvation-induced interactions between two partially hydrophilic sheets}

In this section we consider a more complex model-a hydrophobic sheet containing a few hydrophilic atomic sites. The focus here is on effects of the distribution of hydrophilic sites on the PMFs. One can construct a variety of partially
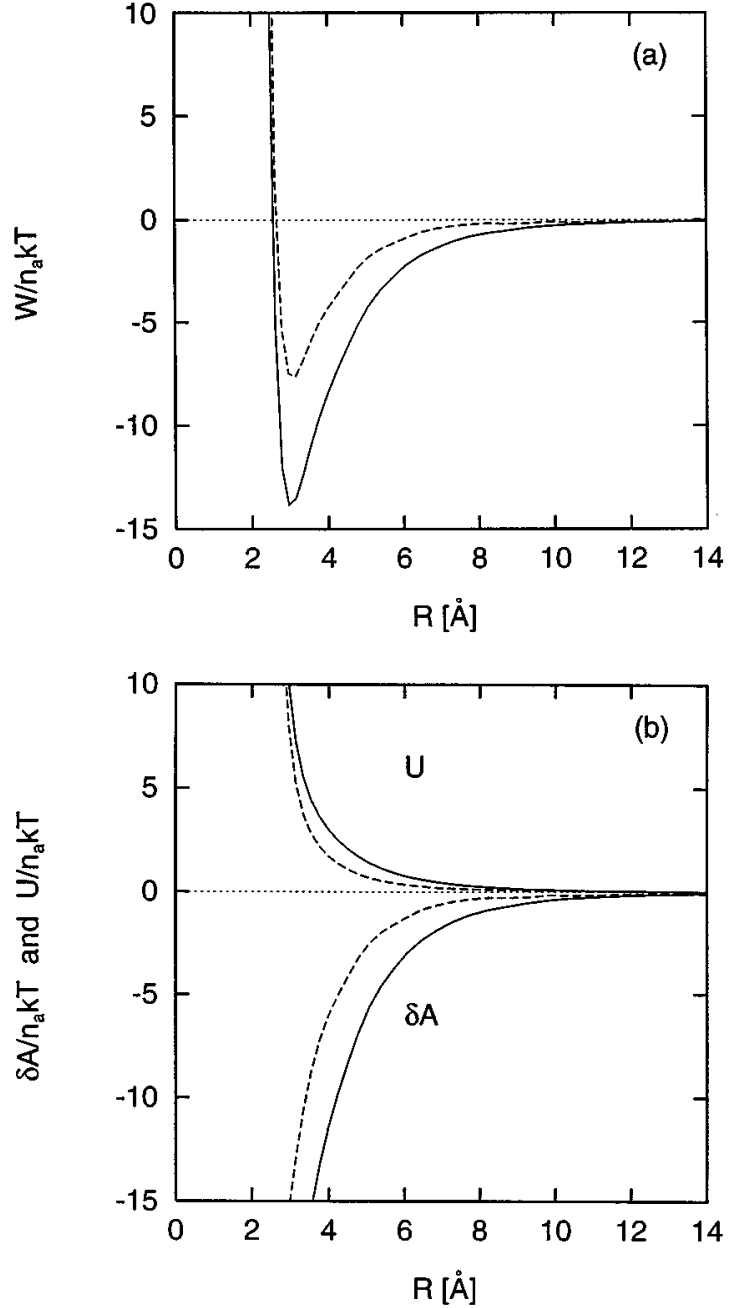

FIG. 8. Comparison of the interactions per atomic site between two hydrophilic molecular sheets of different sizes, $n_{a}=4$ (solid line) and $n_{a}=16$ (dashed line). (a) The PMFs; (b) the direct potential functions and the solvent-induced interactions. Magnitude of the partial charge $|Z|$ is $0.8 \mathrm{e}$. All pairs are in the charge symmetric configuration.

hydrophilic molecular sheets by imposing partial charges on some of the atomic sites on the sheets. In this study we selected two types of $4 \times 4$ partially hydrophilic sheets, shown in Fig. 9. This first type has negative and positive partial charges on the four exterior atomic sites at the corners

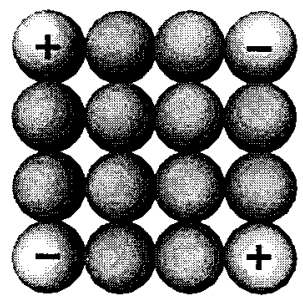

Type I

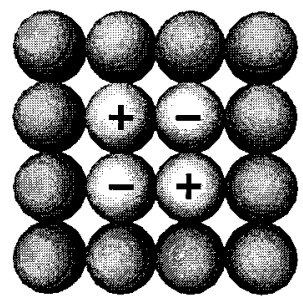

Type II
FIG. 9. Two models of partially hydrophilic molecular sheet: (a) type I, the hydrophilic sites are dispersed at the corner of molecular sheet; and (b) type II, the hydrophilic sites are assembled at the interior region of the molecular sheet. 


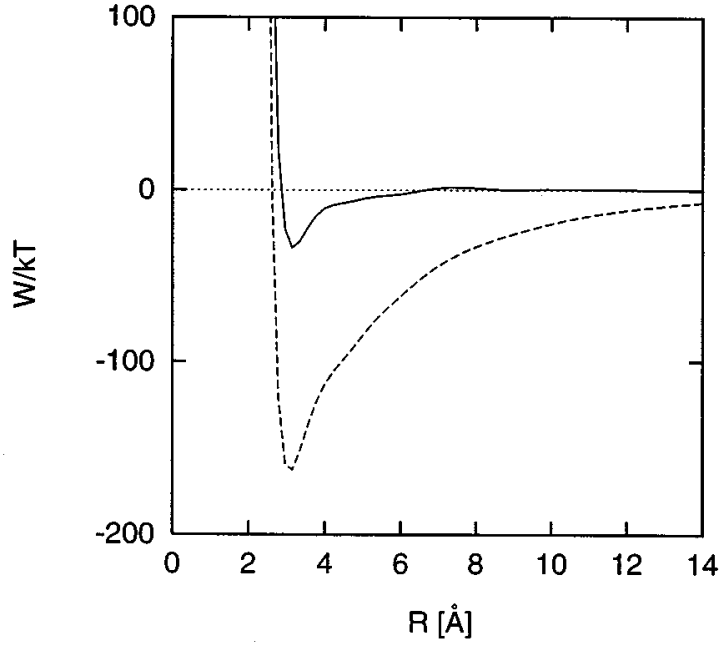

FIG. 10. The PMFs between two type I partially hydrophilic molecular sheets (dashed line) and between two type II partially hydrophilic molecular sheets (solid line); both molecular pairs are in the charge symmetric configuration.

of the sheet while the second type has the charges on the four interior sites. (We hereafter refer to the former sheet as type I and the latter one as type II.) Note that both types of sheet have the same ratio of hydrophilic site number to hydrophobic site number. These two types represent two limiting cases of hydrophilic site distribution; for the type II sheet, the hydrophilic sites are clustered together in a small region, while for the type I sheet, hydrophilic sites are apart from each other.

We compared the PMFs between two molecular sheets for both type I and II sheets in the charge symmetric configuration. Figure 10 shows a large difference between the PMFs of both types. Much stronger attractive interactions are seen between two type I sheets. Moreover because the direct interaction between two type I sheets is stronger and repulsive, the above result indicates the solvent-induced attractive interaction dominates the total interaction between two type I sheets. Note also that since differences between type I and type II sheets can be further divided into (i) the location of hydrophilic sites on a sheet (exterior or interior of the sheet) and (ii) the distance between hydrophilic sites on one molecular sheet (being separated from or close to each other), it would be desirable to separately study the effect on the solvent-induced PMFs due to these two differences. We find it is possible to examine the effect due to the hydrophilic site location difference by calculating the free energy changes in two charging processes: one for a pair of $4 \times 4$ type II sheets and the other for a pair of $2 \times 2$ hydrophilic molecular sheets. The hydrophilic sites on the type II sheets are identical to those on the $2 \times 2$ hydrophilic molecular sheets except that for the former the hydrophilic atomic sites are surrounded by the hydrophobic atomic sites. The effect of sheet-size disparity between the $4 \times 4$ and $2 \times 2$ pairs is not a concern here since we do not compare the solvation free energies of the pairs directly but only the free energy changes in the charging process for each pair; in other words, (a)
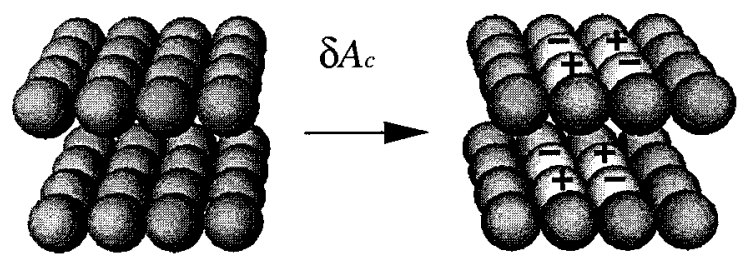

(b)
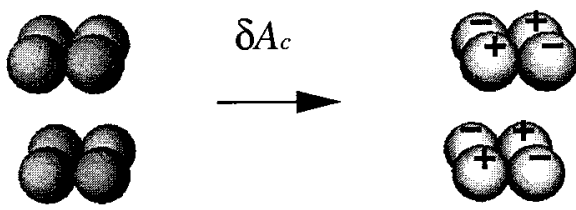

FIG. 11. Two charging processes and the associated solvation free energy changes in water: (a) the charging process for a pair of type II partially hydrophilic molecular sheet; (b) the charging process for a pair of $2 \times 2$ $\left(n_{a}=4\right)$ hydrophilic molecular sheet.

we are only concerned with the solvation free energy change, $\delta A_{c}$, for mutating a pair of hydrophobic sheets to partially or fully hydrophilic sheets (shown in Fig. 11). The free energy changes of the charging process for a pair of sheets in water are plotted as functions of $R$ in Fig. 12. At all distances the two charging processes show almost the same free energy changes. This indicates that the hydrophobic sites which surround the hydrophilic sites have little effect on the solvent-induced interaction. Therefore we can conclude that the large difference of PMFs shown in Fig. 10 is mainly due to the difference in the distribution of hydrophilic sites, and that if the ratio of hydrophilic groups to hydrophobic groups are same, a stronger solvent-induced interaction is expected between two type I partially hydrophilic molecular sheets than between two type II sheets.

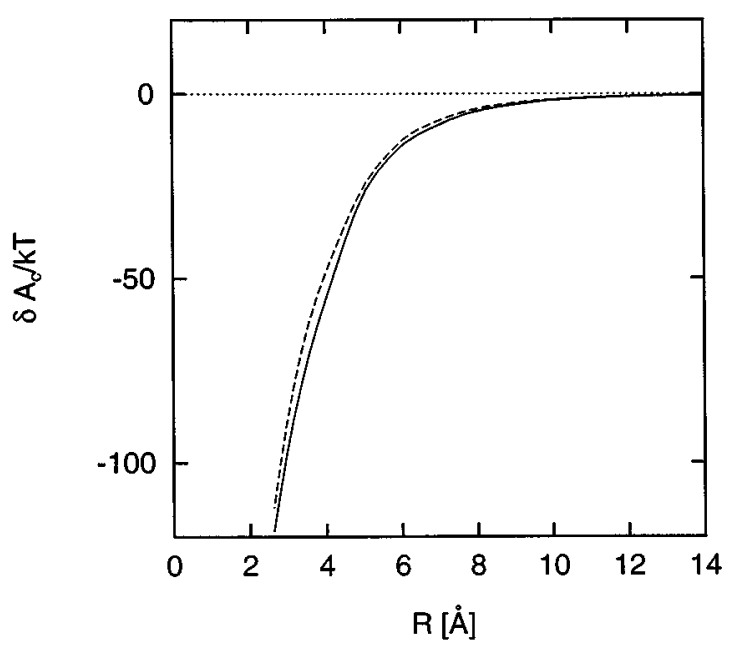

FIG. 12. The solvation free energy of the charging process of two different pairs of molecular sheets shown in Fig. 11: the type II partially hydrophilic molecular sheet (solid line) and the $2 \times 2\left(n_{a}=4\right)$ hydrophilic molecular sheet (dashed line). All pairs are in the charge symmetric configuration. 


\section{E. Components of solvent-induced interaction}

It is well known that the solvent-induced interaction $\delta A$ can be divided into three components: two components associated with the solvation energy differences and one component arising from the solvation entropy difference. It is conceptually helpful to find out the dominant component for both hydrophobic and hydrophilic interactions. Here, specifically, the solvation free energy can be expressed by a sum of the supermolecule-solvent interaction energy, $\Delta E_{\mathrm{uv}}^{*}$, the solvent reorganization energy, $\Delta E_{\mathrm{vv}}^{*}$, and the solvation entropy term, $-T \Delta S^{*}$, i.e.,

$$
\delta A=\delta E_{\mathrm{uv}}+\delta E_{\mathrm{vv}}-T \delta S,
$$

where $\delta Q\left(Q=E_{\mathrm{uv}}, E_{\mathrm{vv}}\right.$, or $\left.S\right)$ is defined as $\Delta Q(\mathbf{R})$ $-\Delta Q(\infty)$. The first term on the right-hand side of Eq. (13) can be determined from the relation

$$
\Delta E_{\mathrm{uv}}^{*}=4 \pi \rho \sum_{i j} \int d r r^{2}\left[g_{i j}^{\mathrm{vu}}(r) u_{i j}^{\mathrm{vu}}(r)\right]
$$

and the third term can be evaluated from the temperature derivative of $\Delta A_{\alpha \beta}^{*}$. Consequently, the solvent reorganization energy difference $\delta E_{\mathrm{vv}}$ can be determined using Eq. (13).

The three components of $\delta A$ for a $4 \times 4$ in-registry pair of hydrophobic molecular sheets are shown in Fig. 13. We find that the solvent-induced potential barrier is mainly due to $\delta E_{\mathrm{uv}}$ and $-T \delta S$ terms, and that effect of the solvent reorganization is relatively small. Furthermore, we find there is no significant difference in all three components of $\delta A$ for water [Fig. 13(a)] and for the hypothetical nonpolar water [Fig. 13(b)].

Figures 14(a) and 14(b) show the three components of $\delta A$ for a $4 \times 4$ pair in the charge symmetric and in the charge asymmetric configurations, respectively. We observe that the strong solvent-induced interactions arise from the $\delta E_{\text {uv }}$ component in both cases and that the magnitude of $\delta E_{\mathrm{uv}}$ is larger than that of $\delta A$ at all distances. We also find that the difference, $\delta A-\delta E_{u v}$, increases as two hydrophilic sheets approach each other. In contrast, contribution of the solvation entropy component, $-T \delta S$, to $\delta A$ is negligibly small, i.e., Eq. (13) can be rewritten as $\delta A-\delta E_{\mathrm{uv}} \sim \delta E_{\mathrm{vv}}$. For a pair of sheets in the charge symmetric configuration, $\delta E_{\mathrm{vv}}$ is positive and increases as two sheets approach each other. Here the solvent reorganization component $\delta E_{\mathrm{vv}}$ effectively plays a role of repulsive interaction between two sheets. On the other hand, for two sheets in the charge asymmetric configuration $\delta E_{\mathrm{vv}}$ leads to effectively attractive interaction between them. The different behaviors of $\delta E_{\mathrm{vv}}$ in two cases can be explained as $\delta E_{\mathrm{vv}}$ has an opposite trend to $\delta E_{\mathrm{uv}}$ and follows the same trend as the direct Coulombic interaction between two sheets [see Figs. 14(a) and 14(b)]. Note that the magnitude of $\delta E_{\mathrm{vv}}$ is found to be larger than the intersheet direct interaction in vacuum but less than $\delta E_{\mathrm{uv}}$. These detailed analyses of the three components of $\delta A$ leads to the conclusion that the controlling component of $\delta A$ is $\delta E_{\mathrm{uv}}$.
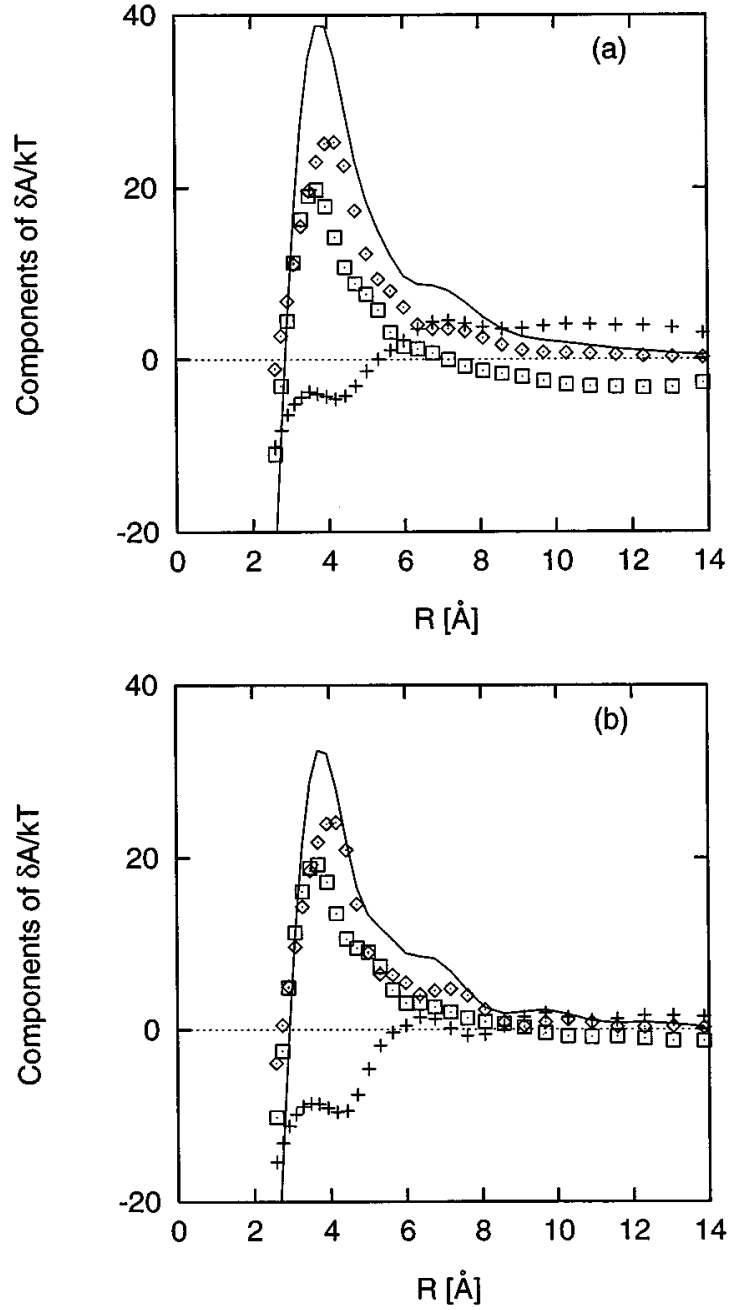

FIG. 13. The three components of the solvent-induced interaction between two hydrophobic molecular sheets $\left(n_{a}=16\right)$ in (a) water and (b) the hypothetical nonpolar water: the supermolecule (the pair of sheet)-solvent interaction energy difference $\delta E_{\mathrm{uv}}(\diamond)$; the solvent reorganization energy difference $\delta E_{\mathrm{vv}}(+)$; and the solvation entropy difference $\delta S(\square)$. The total solvent-induced interaction $\delta A$ (solid line) are also shown. The pair of molecular sheets is in the in-registry configuration.

\section{CONCLUSIONS}

Solvent-induced interactions between hydrophobic, hydrophilic, and partially hydrophilic molecular sheets in water and in the hypothetical nonpolar water were investigated by use of the extended RISM integral equation theory. Through a systematic study for a variety of idealized planar molecular sheets, more insightful results on both hydrophobic and hydrophilic interactions have been attained. We note again that these results are qualitative or semiquantitative in nature due to the inherent approximation in the RISM integral equation theory. The obtained results can be summarized as follows.

(a) The solvation free energy of a single hydrophobic sheet in water depends linearly on the sheet size, which is in good agreement with experimental results of the solvation free energy of hydrocarbons in water.

(b) The PMF per atomic site between two parallel hydrophobic molecular sheets is sensitive to the sheet size. As 

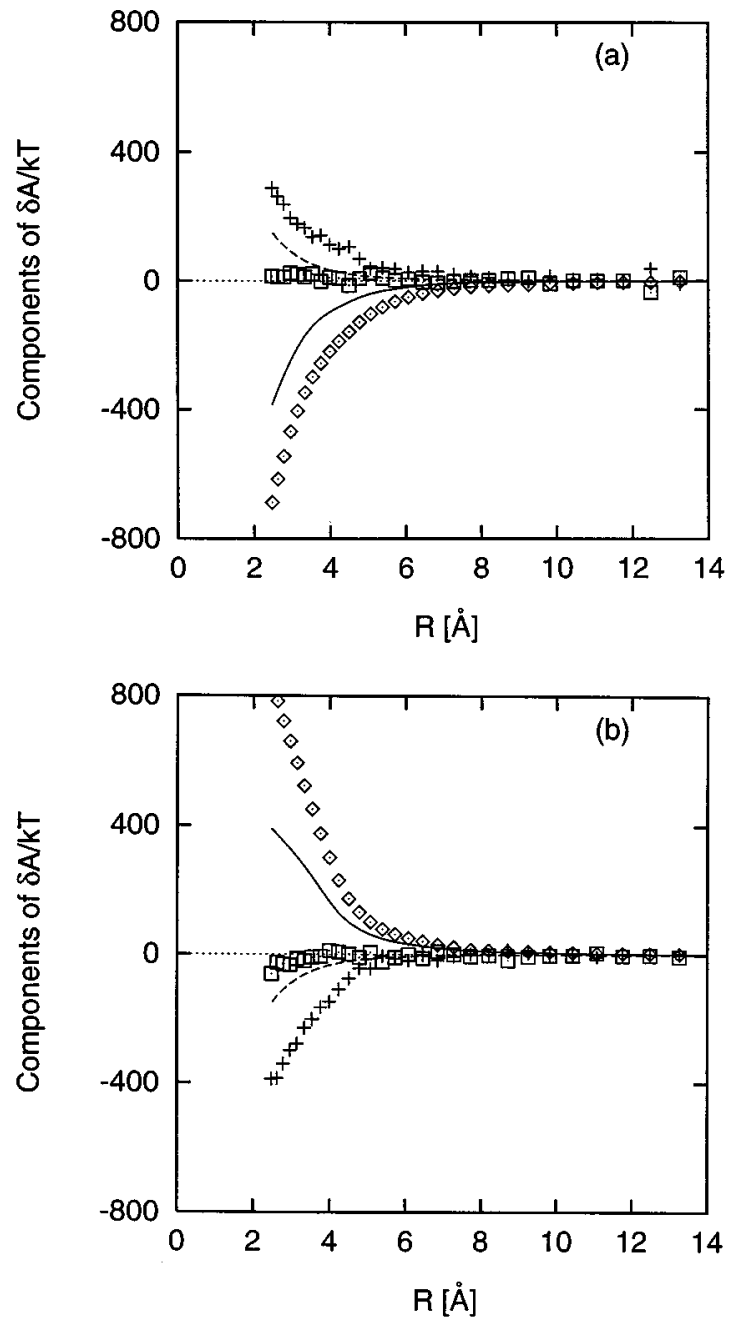

FIG. 14. The three components of the solvent-induced interactions between (a) two charge-symmetric hydrophilic molecular sheets and (b) two chargeasymmetric hydrophilic molecular sheets: the supermolecule (the pair of sheet)-solvent interaction energy difference $\delta E_{\mathrm{uv}}(\diamond)$; the solvent reorganization energy difference $\delta E_{\mathrm{vv}}(+)$; and the solvation entropy difference $\delta S(\square)$. The total solvent-induced interaction $\delta A$ (solid line) and the direct Coulombic interaction $U_{c}$ (dashed line) are also shown. The size of the sheet $n_{a}$ is 16 .

the sheet size increases, the potential barrier separating the contact configuration and the solvent-separated configuration is upraised.

(c) As far as the molecular sheet-size dependence of the solvent-induced interaction is considered no significant difference is found between water and the hypothetical nonpolar water. One possible explanation of this result is that the hydrophobic interaction in water is not as special as usually expected, at least in the infinitely dilute aqueous solution. If this is true, it seems that the hydrophobic attraction (either contact or solvent separated) can be explained as a reflection of the local liquid packing analogous to that shown in the pair distribution function of liquid between two hydrophobic walls. ${ }^{24,54,55}$ One the other hand, the above conclusion should be considered tentative because the pressure of the TIPS water and the hypothetical nonpolar water (at the same density) is very different. (d) In water, the solvent effect on the hydrophilic interaction is substantially larger than that on the hydrophobic interaction. This confirms the conclusion, made by BenNaim from a theoretical estimation of the solvent-induced force using experimental data, ${ }^{43}$ that water affects the interaction between two hydrophilic surfaces much stronger than that between two hydrophobic surfaces. A counter-intuitive feature we find is that the PMF between two hydrophilic sheets is against the direct interaction between two sheets in vacuum.

(e) Solvent-induced interactions between hydrophilic sheets are also very sensitive to the magnitude of partial charge of atomic sites on molecular sheets (a measure of hydrophilicity). A slight change in hydrophilicity may greatly alter characteristics of the hydrophilic interaction between macromolecules in water.

(f) The interaction between two partially hydrophilic sheets in water depends strongly on the distribution of hydrophilic sites on the molecular sheets. With the same number of hydrophilic sites on one sheet a much stronger solvent-induced interaction is found for disperse than clustered hydrophilic site distribution.

Experimental investigations on the solvent-induced interactions between hydrophobic and hydrophilic molecules are needed to confirm the above conclusions. A promising approach is to undertake a force-distance measurement in water by using the atomic force microscopy (AFM) or surface force apparatus (SFA) ${ }^{54,56,57}$ Application of the AFM and SFA has been rapidly growing and, particularly, has been used to image biomolecules in solvents. ${ }^{58-62}$

\section{ACKNOWLEDGMENTS}

We are grateful to Mark Gibson and Joy Woller for their assistance in figure plotting and copy editing, and Dr. D. Diestler and Dr. Y. Zhao for helpful discussions. X. C. Z. is grateful for support from the NSF (GOALI grant) and ONR. Acknowledgment is also made to the donors of the Petroleum Research Fund administered by the American Chemical Society for partial support of this work.

${ }^{1}$ F. Franks, Faraday Symp. Chem. Soc. 17, 7 (1982).

${ }^{2}$ A. Ben-Naim, Water and Aqueous Solutions (Plenum, New York, 1974).

${ }^{3}$ G. Ravishanker and D. L. Beveridge, in Theoretical Chemistry of Biological Systems, edited by G. Naray-Szabo (Plenum, New York, 1986).

${ }^{4}$ J. A. McCammon and S. Harvey, Dynamics of Proteins and Nucleic Acids (Cambridge University Press, Cambridge, 1987).

${ }^{5}$ C. L. Brooks III, M. Karplus, and B. M. Pettitt, in Advances in Chemical Physics (Wiley, New York, 1988), Vol. 71.

${ }^{6} \mathrm{~J}$. N. Israelachvili, Intermolecular and Surface Forces, 2nd ed. (Academic, London, 1992).

${ }^{7}$ M. Levitt and R. Sharon, Proc. Natl. Acad. Sci. U.S.A. 85, 7557 (1988).

${ }^{8}$ T. Lazaridis and M. E. Paulaitis, J. Phys. Chem. 96, 3847 (1992).

${ }^{9}$ F. Hirata and P. J. Rossky, Chem. Phys. Lett. 83, 329 (1981).

${ }^{10}$ F. Hirata, B. M. Pettitt, and P. J. Rossky, J. Chem. Phys. 77, 509 (1982).

${ }^{11}$ D. Chandler and H. C. Andersen, J. Chem. Phys. 57, 1930 (1972).

${ }^{12}$ J. P. Hansen and I. R. MacDonald, Theory of Simple Liquids (Academic, London, 1990).

${ }^{13}$ S. W. Chen and P. J. Rossky, J. Phys. Chem. 97, 6078 (1993).

${ }^{14}$ L. Pratt and D. Chandler, J. Chem. Phys. 67, 3683 (1977).

${ }^{15}$ L. Pratt and D. Chandler, J. Chem. Phys. 73, 3434 (1980).

${ }^{16}$ A. Tani, Mol. Phys. 51, 161 (1984).

${ }^{17}$ H. Tanaka, J. Chem. Phys. 86, 1512 (1987). 
${ }^{18}$ C. Pangali, M. Rao, and B. J. Berne, J. Chem. Phys. 71, 2975 (1979).

${ }^{19}$ S. Swaminathan and D. L. Beveridge, J. Am. Chem. Soc. 101, 5832 (1979).

${ }^{20}$ A. Geiger, A. Rahman, and F. H. Stillinger, J. Chem. Phys. 70, 263 (1979).

${ }^{21}$ S. Okazaki, K. Nakanishi, H. Touhara, and Y. Adachi, J. Chem. Phys. 71, 2421 (1979).

${ }^{22}$ D. C. Rapoport and H. A. Scheraga, J. Phys. Chem. 86, 873 (1982).

${ }^{23}$ G. Ravishanker, M. Mezei, and D. L. Beveridge, Faraday Symp. Chem. Soc. 17, 79 (1982).

${ }^{24}$ C. Y. Lee, J. A. McCammon, and P. J. Rossky, J. Chem. Phys. 106, 4096 (1984).

${ }^{25}$ K. Watanabe and H. C. Andersen, J. Phys. Chem. 90, 795 (1986).

${ }^{26}$ A. Wallqvist, J. Phys. Chem. 95, 8921 (1991).

${ }^{27}$ B. Guillot and Y. Guissani, J. Chem. Phys. 99, 8075 (1993).

${ }^{28}$ D. van Belle, and S. J. Wodak, J. Am. Chem. Soc. 115, 647 (1993).

${ }^{29}$ L. X. Dang, J. Chem. Phys. 100, 9032 (1994).

${ }^{30}$ F. Yoshifumi and M. Suzuki, J. Phys. Chem. 100, 5634 (1996).

${ }^{31}$ M. Berkowitz, O. Karim, J. A. McCammon, and P. J. Rossky, Chem. Phys. Lett. 105, 557 (1984).

${ }^{32}$ A. C. Belch, M. Berkowitz, and J. A. McCammon, J. Am. Chem. Soc. 108, 1755 (1986).

${ }^{33}$ J. Gao, J. Phys. Chem. 98, 6049 (1994)

${ }^{34}$ B. M. Pettitt and P. J. Rossky, J. Chem. Phys. 84, 5836 (1986).

${ }^{35}$ J. Perkyns and B. M. Pettitt, J. Chem. Phys. 97, 7656 (1992).

${ }^{36}$ R. A. Friedman and M. Mezei, J. Chem. Phys. 102, 419 (1995).

${ }^{37}$ L. X. Dang and B. M. Pettitt, J. Am. Chem. Soc. 109, 5531 (1987); J. K. Buckner and W. Jorgensen, ibid. 111, 2507 (1987); H. A. Yu, B. Roux, and M. Karplus, J. Chem. Phys. 92, 5020 (1990); L. X. Dang and B. M. Pettitt, J. Phys. Chem. 94, 4303 (1990); E. Guardia, R. Rey, and J. A. Padro, J. Chem. Phys. 95, 2823 (1991); L. X. Dang, B. M. Pettitt, and P. J. Rossky, ibid. 96, 4046 (1992); O. A. Karim, ibid. 96, 9237 (1992).

${ }^{38}$ M. Matsumoto, H. Tanaka, and K. Nakanishi, J. Chem. Phys. 99, 6935 (1993).

${ }^{39}$ S. R. Durell, B. R. Brooks, and A. Ben-Naim, J. Phys. Chem. 98, 2198 (1994).
${ }^{40}$ B. M. Pettitt, M. Karplus, and P. J. Rossky, J. Phys. Chem. 90, 6335 (1986).

${ }^{41}$ H. A. Yu, B. M. Pettitt, and M. Karplus, J. Am. Chem. Soc. 113, 2425 (1991).

${ }^{42}$ Y. Sun, D. Spellmeyer, D. A. Pearlman, and P. Kollman, J. Am. Chem. Soc. 113, 2425 (1991).

${ }^{43}$ A. Ben-Naim, J. Chem. Phys. 93, 8196 (1990).

${ }^{44}$ A. Ben-Naim, Solvation Thermodynamics (Plenum, New York, 1987).

${ }^{45}$ S. J. Singer and D. Chandler, Mol. Phys. 55, 621 (1985).

${ }^{46}$ D. A. Zichi and P. J. Rossky, J. Chem. Phys. 84, 1712 (1986).

${ }^{47}$ K. Koga, H. Tanaka, and X. C. Zeng, J. Phys. Chem. 100, 16711 (1996).

${ }^{48}$ W. Jorgensen, J. Am. Chem. Soc. 103, 335 (1981).

${ }^{49}$ B. M. Pettitt and P. J. Rossky, J. Chem. Phys. 77, 1451 (1982).

${ }^{50}$ A. Ben-Naim and Y. Marcus, J. Chem. Phys. 81, 2016 (1984)

${ }^{51}$ K. A. Sharp, A. Nicholls, R. F. Fine, and B. Honig, Science 252, 106 (1991).

${ }^{52}$ Here pressure is obtained for the LJ fluid instead of the hypothetical water since no equation of state is available for this model fluid. Pressure for the hypothetical water is expected to be nearly the same as that for the LJ fluid because the difference of the hypothetical water model from the LJ particle is the tinny repulsive core on the the hydrogen atom sites.

${ }^{53}$ J. J. Nicolas, K. E. Gubbins, W. B. Streett, and D. J. Tildesley, Mol. Phys. 37, 1429 (1979).

${ }^{54}$ J. N. Israelachvili and P. M. McGuiggan, Science 241, 795 (1988).

${ }^{55}$ B. Lee, Methods Enzymol. 259, 555 (1995).

${ }^{56}$ H. K. Christenson and C. E. Blom, J. Chem. Phys. 86, 419 (1986).

${ }^{57}$ Y. H. Tsao, D. F. Evans, and H. Wennerström, Science 262, 457 (1993).

${ }^{58}$ H. G. Hansma, M. Bezanilla, F. Zenhausern, M. Adrian, and R. L. Sinsheimer, Nucleic Acids Res. 21, 505 (1993).

${ }^{59}$ Y. L. Lyubchenko, L. S. Shlyakhtenko, R. E. Harrington, P. I. Oden, and S. M. Lindsay, Proc. Natl. Acad. Sci. USA 90, 2137 (1993).

${ }^{60}$ H. G. Hansma, Annu. Rev. Biophys. Biomol. Struct. 23, 115 (1994).

${ }^{61}$ C. Bustamante and D. Keller, Physics Today 48, 32 (1995).

${ }^{62}$ Z. Shao, J. Mou, D. M. Czajkowsky, J. Yang, and J.-Y. Yuan, Adv. Phys. 45, 1 (1996) 